\title{
A Generalized Form of Lait's Modified Potential Vorticity
}

\author{
Rolf MÜller AND GebHard GÜNTHER \\ Institute for Stratospheric Research (ICG I), Research Centre Jülich, Julich, Germany
}

(Manuscript received 6 January 2003, in final form 21 March 2003)

\section{ABSTRACT}

\begin{abstract}
Ertel's potential vorticity $P$ is in widespread use as a diagnostic of dynamical processes in the stratosphere. For a variety of applications, however, the exponential increase of $P$ with altitude is problematic. For this reason, Lait proposed a modified potential vorticity $\Pi_{L}$, where a physically meaningful scaling is introduced that removes much of the altitude dependence of $P$. Here a generalized form of $\Pi_{L}$ is proposed by introducing an additional degree of freedom in the scaling. This generalized modified potential vorticity $\Pi_{g}$ possesses the same conservation properties as $\Pi_{L}$ itself and as the classic potential vorticity $P$ but can be adjusted more closely to the specific situation under investigation. Comparison, over a large altitude range in the stratosphere, of fields of $\Pi_{g}$ with dynamical measures of the polar vortex edge and with observations of the long-lived trace gas $\mathrm{N}_{2} \mathrm{O}$ shows that $\Pi_{g}$ constitutes a more intuitively interpretable quantity than $\Pi_{L}$.
\end{abstract}

\section{Introduction}

Potential vorticity (PV) is a quantity that is in widespread use as a diagnostic of stratospheric dynamics since many years. Its definition is based on the hydrodynamic vorticity equation and the corresponding conservation principle originally formulated by Ertel (1942a,b); it is therefore also commonly referred to as Ertel's potential vorticity. Potential vorticity is frequently used in approximated form for hydrostatic flow on large scales (e.g., Clough et al. 1985):

$$
P=-g\left(\zeta_{\theta}+f\right) \frac{\partial \theta}{\partial p} \text {. }
$$

Here, the relative vorticity $\zeta_{\theta}$ involves differentiation of the horizontal wind components on a given isentropic surface, $g$ is the acceleration of gravity, and the Coriolis parameter $f=2 \Omega \sin \phi$, where $\Omega$ is the angular rotation rate of the earth and $\phi$ latitude. Further, $p$ is pressure and $\theta$ is the potential temperature, defined as

$$
\theta=T\left(\frac{p_{0}}{p}\right)^{R / c_{p}}=T\left(\frac{p_{0}}{p}\right)^{\kappa}
$$

where $R$ is the gas constant for dry air, $c_{p}$ the specific heat at constant pressure $\left(\kappa=R / c_{p}\right), T$ the temperature, and $p_{0}$ a reference pressure (1000 $\mathrm{hPa}$ for the calculations below).

For the large-scale circulations typical for the strato-

Corresponding author address: Dr. Rolf Müller, Forschungszentrum Jülich, Institute for Stratospheric Research (ICG I), $52425 \mathrm{Ju}-$ lich, Germany.

E-mail: ro.mueller@fz-juelich.de, E-mail: g.guenther@fz-juelich.de sphere, which are characterized by large Richardson numbers, PV may be approximated as (e.g., Hartmann 1977; Butchart and Remsberg 1986)

$$
P=-g \zeta_{a} \frac{\partial \theta}{\partial p}
$$

where $\zeta_{a}=\zeta_{p}+f$ is the component of the absolute vorticity perpendicular to an isobaric surface and $\zeta_{p}$ involves differentiation of the horizontal wind components on constant pressure levels.

Potential vorticity is materially conserved in the absence of friction and diabatic heating, so that on short enough timescales, of the order of days in most parts of the atmosphere, it may be considered as a tracer of fluid motion (see, e.g., McIntyre 1992). For example, Leovy et al. (1985) have shown that for the winter polar stratosphere fields of PV and ozone (which may be considered a quasi-conservative tracer under these conditions) show good agreement. Fields of potential vorticity have also successfully been employed to investigate the temporal development of the polar vortices (e.g., Butchart and Remsberg 1986; Manney et al. 1996; Manney et al. 1999; Günther et al. 2002). Further, because the boundary of the polar vortex is characterized by the maximum gradient in PV on an isentropic surface (e.g., McIntyre 1992), this quantity may be used as a diagnostic tool to describe the location of the vortex edge.

Moreover, potential vorticity is used as a "coordinate" containing information on stratospheric dynamics to map tracer fields with limited spatial coverage (e.g., from observations or from two-dimensional models) to the three-dimensional fields required for the initialization of three-dimensional chemistry transport models 
(e.g., Lait 1994; Lary et al. 1995; McKenna et al. 2002b).

One problem arising from the definition of the PV [Eqs. (1) and (3)] is its exponential increase with altitude due to the term $\partial \theta / \partial p$. This renders difficult both comparing horizontal distributions at different altitudes and interpreting vertical cross sections of PV in the middle atmosphere. Moreover, the use of $\theta$ and PV as independent variables for tracer reconstruction (Schoeberl et al. 1992) is complicated by the increase of PV with altitude.

To remedy this problem, Dunkerton and Delisi (1986) suggested scaling PV with a factor $-\left[g \partial \theta_{\mathrm{sa}} / \partial p\right]^{-1}$ where $\partial \theta_{\mathrm{sa}} / \partial p$ is calculated from the U.S. Standard Atmosphere, 1976 (USSA). Such a scaling makes it easier to compare potential vorticity maps on different potential temperature surfaces and changes the units of the scaled PV to notional vorticity units (per second). This type of scaling has been used in several studies (e.g., Manney et al. 1994, 1996; Manney et al. 1999; Dameris et al. 1995; Rex et al. 1998).

A different approach is put forward by Kouker et al. (1999) who employ a vertical scaling factor of $\exp \left(-z_{\mathrm{sa}} / H\right)$, with $H$ being the scale height $(7 \mathrm{~km})$ and $z_{\mathrm{sa}}$ the pressure altitude of the corresponding isentropic surface of the USSA. Alternatively, because $z_{\mathrm{sa}}=-H$ $\ln \left(p_{\mathrm{sa}} / p_{0}\right)$, where $p_{\mathrm{sa}}$ is the pressure on the isentropic surface of the USSA in question, the scaling factor may be expressed as $p_{\mathrm{sa}} / p_{0}$, or, in a more general formulation, as $p_{\mathrm{sa}} / p_{\text {ref }}$, where $p_{\text {ref }}$ is the reference pressure level, for which the scaling corresponds to unscaled potential vorticity units. Because the scale factor is dimensionless, this scaling preserves the unit of potential vorticity. Further, because for both the Kouker et al. (1999) and the Dunkerton and Delisi (1986) scaling the PV on a given isentropic surface is multiplied by a constant factor (that is deduced from parameters of the USSA corresponding to this isentropic surface), the structure of PV on a given isentropic surface is preserved by these two scalings.

Lait (1994) proposed a modified potential vorticity $\Pi_{L}$ defined as Ertel's potential vorticity multiplied by a scaling factor $\left(\theta / \theta_{0}\right)^{-9 / 2}$, where $\theta_{0}$ is a constant to make the scaling factor dimensionless. This scaling describes the vertical gradient of $\theta$ for an isothermal atmosphere and therefore removes much of the altitude dependence of PV, without destroying the structure of PV on a given isentropic surface. Moreover, this scaling preserves both the unit and the conservation properties of PV.

Modified potential vorticity $\Pi_{L}$ fields have been used for a variety of problems, for example, to analyze meteorological conditions in support of satellite (Günther et al. 2002) and airborne (Morgenstern and Carver 2001) measurements or to investigate the structure of the polar vortices (Waugh and Randel 1999; Polvani and Saravanan 2000; Greenblatt et al. 2002). Further, $\Pi_{L}$ is useful for the initialization of chemical models (Lait 1994; Schoeberl et al. 1996).
However, above $\approx 30 \mathrm{hPa}(25 \mathrm{~km}, 650 \mathrm{~K}) \Pi_{L}$ values usually decrease with altitude in the high latitudes in winter (Lait 1994; Günther et al. 2002), an observation that could be erroneously interpreted as a decrease of the vortex strength with altitude. Further, this property hampers the use of $\Pi_{L}$ as a quasi-conserved coordinate in the analysis of chemical constituent data or as a tool for chemical model initialization.

To obviate such problems, we propose here a generalized form of Lait's (1994) modified PV $\Pi_{L}$ by introducing an additional degree of freedom by allowing a nonisothermal vertical scaling. We suggest that fields of this generalized modified $\mathrm{PV} \Pi_{g}$, if considered as vertical maps, describe more intuitively the vertical structure of the polar vortex air mass and are in better agreement with measured fields of long-lived trace compounds. The $\Pi_{g}$ may therefore serve as a diagnostic tool to produce fields of the vortex structure that are more easily interpreted than fields produced from other forms of potential vorticity.

\section{Generalization of Lait's modified potential vorticity}

\section{Nonisothermal scaling}

In the following we will consider potential vorticity $P$ on surfaces of constant pressure [Eq. (3)]. Lait (1994) has shown that for an isothermal atmosphere $P$ varies with pressure as $\approx \theta^{9 / 2}$ and has thus suggested to scale $P$ by a dimensionless factor, $\left(\theta / \theta_{0}\right)^{-9 / 2}$.

Here we propose a similar but generalized scaling that allows for temperature variation with altitude and thereby introduces an additional degree of freedom. Assume a temperature profile dependent on pressure $p$ of the form

$$
T(p)=T_{0}\left(\frac{p_{0}}{p}\right)^{\alpha},
$$

where $T_{0}$ is a constant reference temperature and $\alpha$ is a dimensionless parameter that may be chosen so that the resulting temperature profile is reasonably realistic for the meteorological situation in question. The reference temperature $T_{0}$ is the temperature at the reference pressure $p_{0}$ [cf. Eq. (2)]. Figure 1 shows an example of a temperature profile with $T_{0}=187 \mathrm{~K}, p_{0}=1000 \mathrm{hPa}$, and $\alpha=1 / 21$ to be used below. Combining Eqs. (2) and (4) permits writing

$$
\theta=T_{0}\left(\frac{p_{0}}{p}\right)^{\alpha+\kappa}
$$

and, taking the derivative of Eq. (5) with respect to $p$,

$$
\frac{\partial \theta}{\partial p}=-\frac{(\alpha+\kappa) T_{0}}{p_{0}}\left(\frac{p_{0}}{p}\right)^{\alpha+\kappa+1} .
$$

Then, writing 


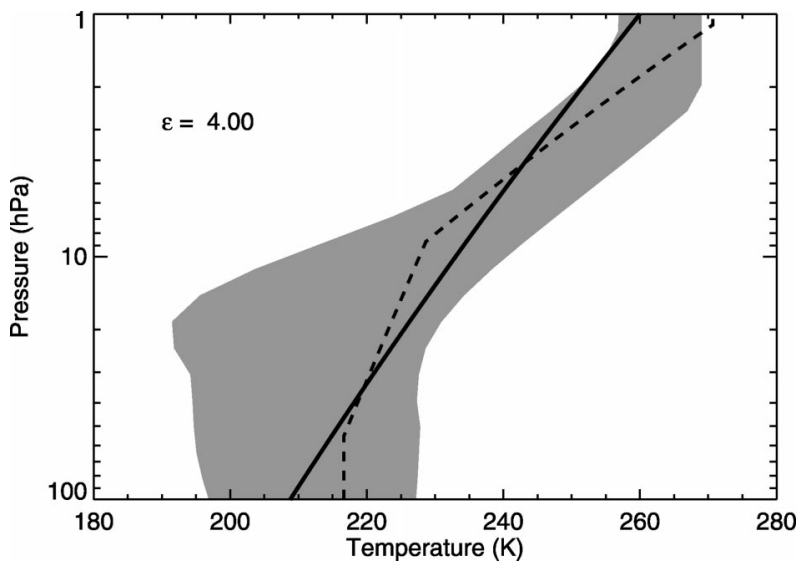

FIG. 1. Example of an analytical temperature profile (solid line) with $\alpha=1 / 21, \varepsilon=4.0, p_{0}=1000 \mathrm{hPa}$, and $T_{0}=187 \mathrm{~K}$ [cf. Eq. (4)]. Also shown for comparison is the temperature profile of the USSA (dashed line) and the range of temperatures in the COSPAR International Reference Atmosphere (Chandra et al. 1990) between $-70^{\circ}$ and $70^{\circ}$ lat for Aug (gray shaded area).

$$
\frac{\partial \theta}{\partial p}=-\frac{\alpha+\kappa}{p_{0}} T_{0}^{1-\varepsilon}\left[T_{0}\left(\frac{p_{0}}{p}\right)^{\alpha+\kappa}\right]^{\varepsilon},
$$

where

$$
\varepsilon=\frac{(\alpha+\kappa+1)}{(\alpha+\kappa)},
$$

(and $\alpha=1 /(\varepsilon-1)-\kappa$ ) shows that $\partial \theta / \partial p$ depends on $\theta$ as

$$
\frac{\partial \theta}{\partial p} \propto \theta^{\varepsilon}
$$

The parameter $\varepsilon$, referred to as "polytropic exponent" hereafter, depends on the assumed temperature profile. For example, for an isothermal atmosphere, $\alpha=0$ and $\varepsilon=(\kappa+1) / \kappa=9 / 2$ so that

$$
\frac{\partial \theta}{\partial p} \propto \theta^{(\kappa+1) / \kappa}=\theta^{9 / 2},
$$

which is the case discussed by Lait (1994). Following his line of arguments, we propose a generalized form of modified potential vorticity

$$
\Pi_{g}=P\left(\frac{\theta}{\theta_{0}}\right)^{-(\alpha+\kappa+1) /(\alpha+\kappa)}=P\left(\frac{\theta}{\theta_{0}}\right)^{-\varepsilon},
$$

where $\theta_{0}$ is a constant ( $475 \mathrm{~K}$ in the examples below) introduced to make the scale factor dimensionless. Note that for positive values of $\alpha$ (i.e., for $\varepsilon<4.5$ ) temperature is increasing with altitude whereas for negative $\alpha$ (i.e., for $\varepsilon>4.5$ ) it is decreasing. The parameter $\varepsilon$ may be chosen depending on the average temperature profile for the meteorological conditions under consideration. In the stratospheric examples discussed below, we obtain a good description of the meteorological situation by choosing $\alpha=1 / 21$ so that $\varepsilon=4$ (Fig. 1) and thus

$$
\Pi_{g}=P\left(\frac{\theta}{\theta_{0}}\right)^{-4} .
$$

The same scaling $(\varepsilon=4)$ was used by Nakamura (1995) in his formulation of a mass equivalent latitude that serves as a meridional coordinate (see also section 4 below).

Lait (1994) demonstrated that for the modified PV $\left(\Pi_{L}\right)$, a conservation property can be derived [Eq. (7) in Lait (1994)] that is equivalent to the material conservation property of potential vorticity $P$ (Haynes and McIntyre 1990; McIntyre 1992; Lait 1994). The fact that the conservation properties of $P$ can be transfered to $\Pi_{L}$ depends on the scale factor's dependence on $(\theta /$ $\left.\theta_{0}\right)^{-\varepsilon}$ but not on the precise value of $\varepsilon=9 / 2$. Therefore, the conservation properties of $P$ can be transfered to $\Pi_{g}$. Further, Lait (1994) has shown that the material time rate of change of $\Pi_{L}$ [his Eq. (8)] may be expressed in a similar manner to that valid for $P$, except for an additional term $-\varepsilon \Pi Q / \theta$, where $Q$ is the heating rate. For $\varepsilon=9 / 2$, this term is not negligible but does not radically change the conservation of $\Pi_{L}$ in comparison with $P$ over timescales for which the changes of $P$ due to diabatic effects can be neglected (Lait 1994). In the examples below, we have chosen $\varepsilon=4<9 / 2$ so that the conservation properties of $\Pi_{g}$ are comparable to $\Pi_{L}$.

\section{Example of an application: Estimating the location of the polar vortex edge}

To illustrate the usefulness of the concept of generalized potential vorticity $\Pi_{g}$, we employ $\Pi_{g}$ here as a diagnostic tool to describe the structure of the polar vortex and to deduce an estimate of the location of the polar vortex edge. Vortex edge is the term commonly used to refer to the location of the transport barrier between the air masses inside and outside of the vortex.

Owing to the existence of this transport barrier (and because of the strong diabatic descent within the vortex), greatly differing mixing ratios of long-lived trace gases (with sufficiently large vertical gradients) are observed inside and outside of the vortex. Examples of such trace gases are $\mathrm{N}_{2} \mathrm{O}, \mathrm{CH}_{4}, \mathrm{HF}, \mathrm{CFC}-11$, and CFC-12. Consequently, measurements of such substances in aircraft transects through the vortex edge clearly show very sharp gradients (e.g., Tuck 1989; Greenblatt et al. 2002; Konopka et al. 2003). The sharp gradient in trace species at the vortex edge is likewise noticeable in satellite measurements (e.g., Manney et al. 1999; Riese et al. 1999). Owing to the presence of large amounts of volcanic aerosol in the stratosphere due to the eruption of Mt. Pinatubo in June 1991, the boundary of the Arctic vortex could also be identified as a strong gradient in aerosol particle surface during the early 1990s (Dameris et al. 1995).

Moreover, the polar vortex edge is characterized by a steep gradient in PV (e.g., McIntyre 1992). Nash et al. (1996) have developed a criterion to determine the 
edge of the vortex based on the maximum gradient of PV in equivalent latitude. Greenblatt et al. (2002) have shown that steep gradients of PV and of chemical tracers are generally collocated at the vortex edge, but also that smaller-scale vortex edge features might not be properly represented in analyses based on PV derived from meteorological fields.

Furthermore, theoretical concepts to describe the location of the polar vortex edge have been put forward such as the location of hyperbolic and elliptical regions in the flow (Koh and Legras 2002) or the location of minimum values of the finite-time Lyapunov exponent (McKenna et al. 2002a; Koh and Legras 2002). Nakamura $(1995,1996)$ considered the mass enclosed by a tracer isosurface as a Lagrangian "meridional" coordinate. In this formalism the "effective diffusivity," a measure of the geometric structure of a tracer field, is defined that allows transport barriers to be located; the polar vortex edge is characterized then as the area of minimum values in effective diffusivity (Nakamura 1996; Haynes and Shuckburgh 2000; Allen and Nakamura 2001).

However, in studies of the polar vortex structure, often a specific value (or a range of values) of PV is taken at a given level of potential temperature as an indication of the steepest gradient in PV and thus as measure of the location of the vortex edge (e.g., Rummukainen et al. 1994; Manney et al. 1994; Dameris et al. 1995; Rex et al. 1998; Günther et al. 2002). While being attractive for practical applications because it allows a clear, simple definition, applying such a procedure of course involves a certain approximation. It is always necessary to verify that this approximation is justified for the particular problem in question. Further, one has to take into account that the approximation will be increasingly inaccurate if used to follow the evolution of the vortex edge over a prolonged period of time. This is so because the change of gradients in PV with time will be different from the change of PV itself (Manney et al. 1994).

\section{a. Scaled potential vorticity versus the Nash criterion as an estimate of the location of the vortex edge}

Often, the location of the edge of the polar vortex is sought over a certain altitude range, that is, over a range of isentropic levels. One possibility is, for all isentropic levels in question, to perform an analysis of the location of the polar vortex edge, for example, based on the maximum gradient of PV (Nash et al. 1996). A simpler alternative, which may be sufficiently accurate for certain applications, is to define the value of PV at the vortex edge for a particular isentropic level and use this value as the edge criterion in a scaled PV space (e.g., Rex et al. 1998; Manney et al. 1999; Polvani and Saravanan 2000; Günther et al. 2002). Such a criterion will therefore be independent of altitude, although, if converted to a PV value, it will result in vortex boundary PV values that increase with altitude. The precise alti-

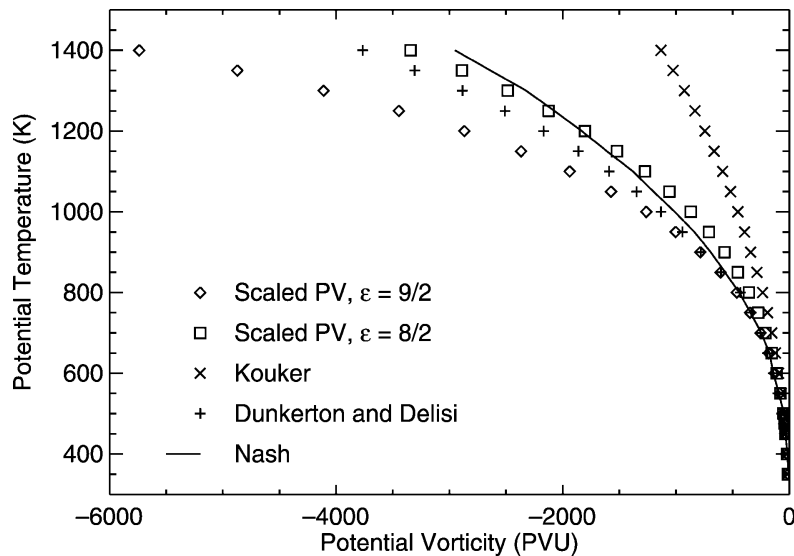

FIG. 2. The value of PV at the edge of the polar vortex on 15 Aug 1997 from UKMO analyses. Diamonds show the vortex edge for modified PV $\Pi_{L}=-44.3 \mathrm{PVU}(\varepsilon=9 / 2)$, squares the edge for generalized modified PV $\Pi_{g}=-44.3 \mathrm{PVU}(\varepsilon=4)$. [1 PVU $=10^{-6}$ $\mathrm{K} \mathrm{m}^{2}\left(\mathrm{~kg} \mathrm{~s}^{-1}\right.$.] Plus signs and crosses indicate the vortex edge deduced using the Dunkerton and Delisi (1986) and the Kouker et al. (1999) scaling, respectively. Also shown, for comparison, is the vortex edge according to the Nash et al. (1996) method (solid line). See text for further details.

tude variation of the PV vortex boundary values depends on the specific choice mode for the scaling of potential vorticity, that is, in the formulation discussed here, on the specific choice of $\varepsilon$ [see Eq. (11)].

As a specific example, we consider the boundary of the Antarctic vortex on 15 August 1997 based on the Met Office (UKMO) analyses. We calculated the PV value at the vortex boundary on $475 \mathrm{~K}$ according to the Nash et al. (1996) method [-44.3 PVU; 1 PVU $=10^{-6}$ $\mathrm{K} \mathrm{m}^{2}(\mathrm{~kg} \mathrm{~s})^{-1}$. This value was then used as the vortex boundary criterion over the altitude range 350-1400 K using modified potential vorticity $\Pi_{L}$ with $\varepsilon=9 / 2$ and generalized modified potential vorticity $\Pi_{g}$ with $\varepsilon=4$; the resulting PV vortex edge values are shown in Fig. 2 (diamonds and squares, respectively). These two vortex edge estimates are compared for 350-1400 K with the vortex edge according to the Nash et al. (1996) method (solid line in Fig. 2). Similarly, the value of $-44.3 \mathrm{PVU}$ at $475 \mathrm{~K}$ can be used as the vortex boundary criterion using the scaling suggested by Kouker et al. (1999; crosses in Fig. 2) and Dunkerton and Delisi (1986; plus signs in Fig. 2). The Kouker et al. (1999) scaling may be applied in the same manner as modified potential vorticity. It leads to considerably lower absolute values of PV than the Nash et al. (1996) criterion as an estimate of the polar vortex edge. However, the Kouker et al. (1999) scaling essentially assumes $\partial \theta / \partial p$ $\propto p_{0} / p$ whereas the Lait (1994) scaling implies $\partial \theta / \partial p \propto$ $\left(p_{0} / p\right)^{\kappa+1}$ with $\kappa+1 \approx 1.3$ [see Eq. (6) above]. If a scaling of the form $\left(p_{0} / p\right)^{1.3}$ were employed (not shown), a much better agreement with the Nash et al. (1996) values would be achieved.

A value of $-44.3 \mathrm{PVU}$ at $475 \mathrm{~K}$ corresponds to a value of $-3.1 \mathrm{~s}^{-1}$ for Dunkerton and Delisi (1986) scal- 
ing; if, similar as above, this value is used as the vortex boundary criterion and converted into a PV value, the resulting vortex boundary estimate leads to a similar pattern as the $\Pi_{g}$ scaling, but with a tendency to somewhat greater absolute values as an estimate of the vortex boundary above $\approx 1100 \mathrm{~K}$.

In the altitude range $350-600 \mathrm{~K}$, differences between the five vortex boundary definitions are relatively small. Between $\approx 600$ and $850 \mathrm{~K}$, the vortex boundary defined using $\Pi_{L}$, that is, $\varepsilon=4.5$, (diamonds in Fig. 2), agrees somewhat better with the Nash et al. (1996) method (solid line) than the vortex boundary defined using $\Pi_{g}$ with $\varepsilon=4.0$ (squares). The Dunkerton and Delisi (1986) scaling (plus signs) gives rather similar results to the $\Pi_{L}$ scaling (diamonds), whereas the Kouker et al. (1999) scaling (crosses) shows a tendency to lower absolute values of PV than the Nash et al. (1996) criterion from $\approx 650 \mathrm{~K}$ on. At greater altitudes (above $\approx 1000 \mathrm{~K}$ ) the values derived based on $\Pi_{L}$ (diamonds) and the Kouker et al. (1999) scaling deviate most strongly from the values deduced from the Nash et al. (1996) method (solid line).

\section{b. Spaceborne tracer observations of the Antarctic polar vortex}

As a further test of the generalized potential vorticity $\Pi_{g}$ as a diagnostic tool to describe the structure of the polar vortex, we consider spaceborne measurements of the long-lived trace gas $\mathrm{N}_{2} \mathrm{O}$ in the stratosphere made by the Cryogenic Infrared Spectrometers and Telescopes for the Atmosphere (CRISTA) instrument (Offermann et al. 1999; Riese et al. 1999) in August 1997. The CRISTA experiment provides data in comparably good horizontal $(\approx 200 \mathrm{~km})$ and vertical $(2-3 \mathrm{~km})$ resolution with almost global coverage. CRISTA measurements of a long-lived tracer like $\mathrm{N}_{2} \mathrm{O}$ are thus particularly well suited for studying the precise location of dynamical structures characterized by strong tracer gradients such as the edge of the polar vortex.

The Antarctic polar vortex is clearly visible in a vertical cross section of CRISTA $\mathrm{N}_{2} \mathrm{O}$ measurements in the Southern Hemisphere along a specific longitude $\left(20^{\circ} \mathrm{W}\right)$ as a region of low $\mathrm{N}_{2} \mathrm{O}$ mixing ratios (Fig. 3, top). The strongest horizontal gradient in $\mathrm{N}_{2} \mathrm{O}$, a measure of the vortex boundary (Greenblatt et al. 2002), is located at $\approx 54^{\circ} \mathrm{S}$ and extends up to the top altitude of the CRISTA measurements. Clearly, there is no indication that the location of the $\mathrm{N}_{2} \mathrm{O}$ gradient, and thus the location of the vortex edge, varies substantially with altitude. The same conclusion would be reached if the horizontal gradient of either $\Pi_{L}$ or $\Pi_{g}$ would be employed as a measure of the vortex edge (Fig. 3, middle and bottom). However, considering a given value of modified $\mathrm{PV} \Pi_{L}$ (with $\varepsilon=9 / 2$; Fig. 3, middle) could lead one erroneously to conclude that the vortex strength is decreasing from 800 to $1100 \mathrm{~K}$ (as the $\Pi_{L}$ contours are bending poleward with altitude). This applies to a much lesser extent to the generalized modified PV $\Pi_{g}$ field with $\varepsilon=4$ (Fig. 3 , bottom), which thus makes $\Pi_{g}$ a more easily interpretable diagnostic tool. However, it cannot be expected that fields of $\mathrm{N}_{2} \mathrm{O}$ mixing ratios resemble very closely the fields of modified PV, owing to the fact that $\mathrm{N}_{2} \mathrm{O}$ mixing ratios in the stratosphere decrease with altitude. This decrease is primarily due to photolytic destruction of $\mathrm{N}_{2} \mathrm{O}$, which is increasing with altitude and is most clearly noticeable in the latitude range $30^{\circ}-10^{\circ} \mathrm{S}$.

Further, we consider horizontal cross sections of CRISTA $\mathrm{N}_{2} \mathrm{O}$ measurements at $\theta=1100$ and $800 \mathrm{~K}$ valid for the last day of measurements, 15 August 1997, 1200 UTC. These were constructed by trajectory mapping (Morris et al. 1995), that is, by calculating forward trajectories to 15 August 1997, 1200 UTC from all CRISTA measurement locations in the earlier measurement period since 8 August 1997 and then mapping forward the CRISTA $\mathrm{N}_{2} \mathrm{O}$ measurements along those trajectories to 15 August 1997, 1200 UTC. Such a mapping is feasible since $\mathrm{N}_{2} \mathrm{O}$ is long lived enough to be considered chemically inert over the time period of a few days. In this way, one obtains a spatial resolution and a coverage even better than the pure measurements.

The vortex edge (and likewise the subtropical mixing barrier) is clearly noticeable as a strong gradient in $\mathrm{N}_{2} \mathrm{O}$ mixing ratios in isentropic maps constructed in this way. Obviously, there is no indication of a weaker vortex at greater altitudes $(\approx 1100 \mathrm{~K}$; Fig. 4 , top) than at lower altitudes ( $\approx 800 \mathrm{~K}$; Fig. 4 , bottom).

For the 800-K level (Fig. 4, bottom) the vortex boundary defined by the Nash et al. (1996) criterion (red line) and the vortex boundary deduced from assuming a constant value of $\Pi_{L}(\varepsilon=4.5)$ or $\Pi_{g}(\varepsilon=4)$ of -44.3 PVU (black and yellow lines, respectively) show an approximate agreement with the vortex edge deduced from the location of the strongest $\mathrm{N}_{2} \mathrm{O}$ gradient in the CRISTA measurements. For the 1100-K level, however, deducing the vortex boundary from the assumption that $\Pi_{L}=-44.3$ PVU (black line in Fig. 4, top) leads to a substantial underestimate of the size of the polar vortex and to a vortex boundary that is clearly located too far poleward.

On both the 800 - and the $1100-\mathrm{K}$ level, the maximum gradient of the CRISTA $\mathrm{N}_{2} \mathrm{O}$ field is located equatorward of a vortex boundary defined on the basis of PV, both according to the Nash et al. (1996) criterion and to choosing a constant value of $\Pi_{g}$. This difference is also evident in the latitude-altitude cross sections shown in Fig. 3. A similar mismatch has been noted by Greenblatt et al. (2002) based on airborne, high-altitude measurements of $\mathrm{N}_{2} \mathrm{O}$ in the Arctic vortex on 27 January 2000 and by Dameris et al. (1995) based on lidar measurements of stratospheric aerosol in winter 1993 and 1994. In both studies this mismatch is considered to be likely due to the limited resolution of the measurement network on which the PV calculations are ultimately based. It is further noticeable in Fig. 4 is that the gradient in $\mathrm{N}_{2} \mathrm{O}$ at the vortex edge is enhanced by tongues of 


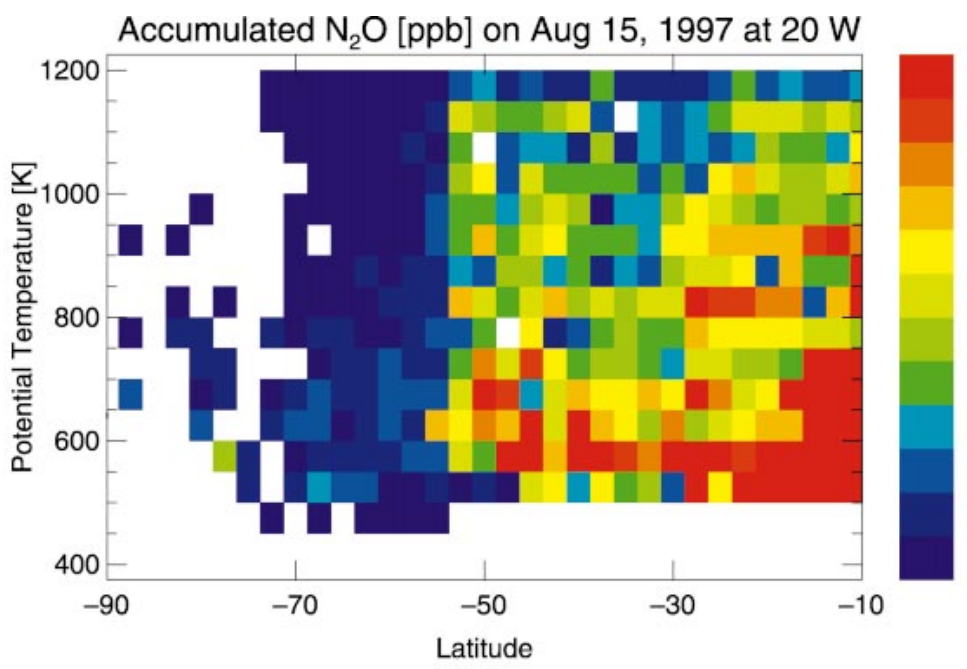

368.41

110.00

100.00

90.00

80.00

70.00

60.00

50.00

40.00

30.00

20.00

10.00

0.00
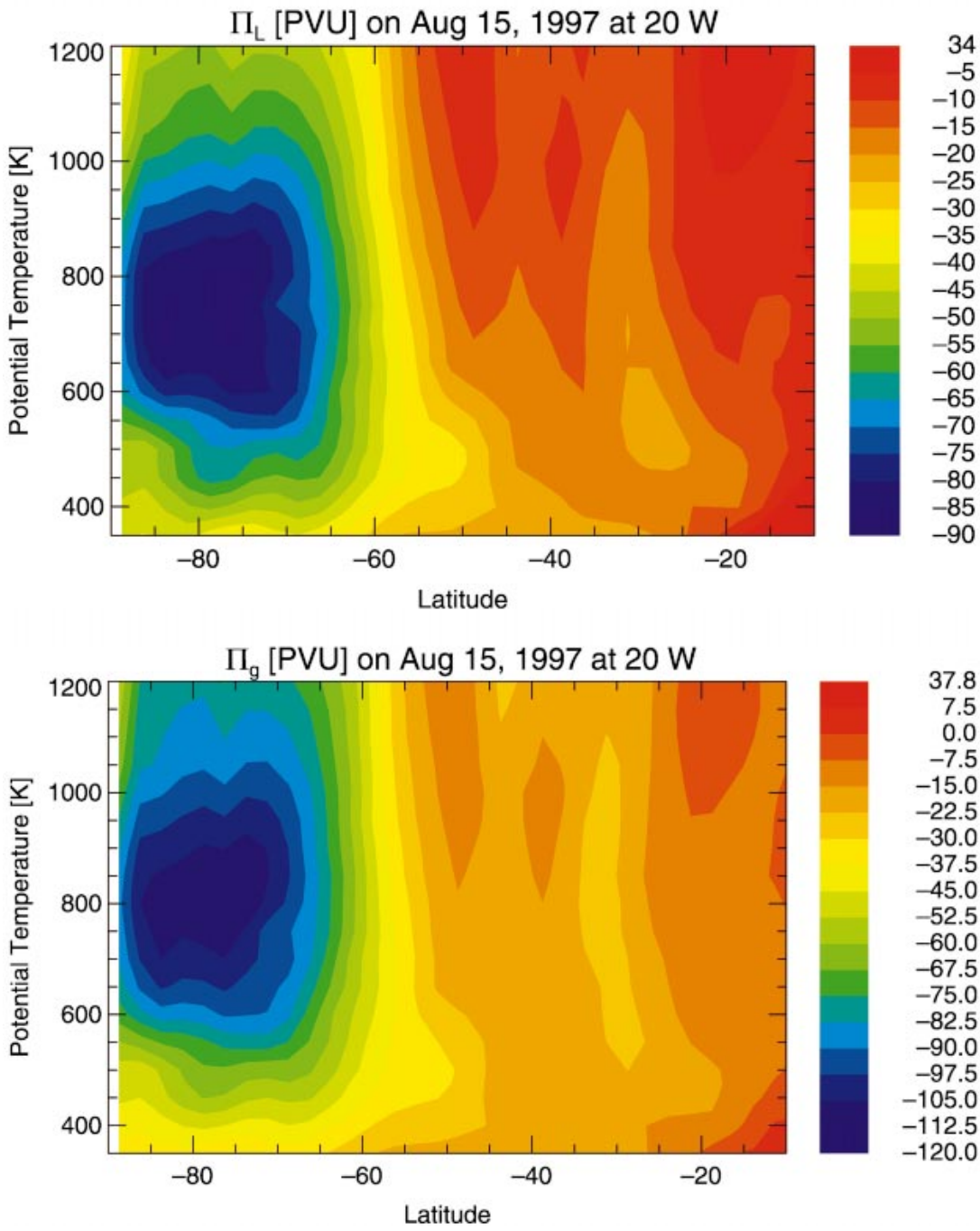

FIG. 3. Latitude-altitude cross section (along $20^{\circ} \mathrm{W}$ long) through the Antarctic polar vortex on 15 Aug 1997: (top) CRISTA measurements of nitrous oxide ( $\mathrm{N}_{2} \mathrm{O}$ ); (middle) Lait's (1994) modified PV $\Pi_{L}$; (bottom) the generalized form of modified PV $\Pi_{g}$ using $\varepsilon=4$ [see Eq. (11)]. (top) The CRISTA measurements were accumulated over a range of $\pm 5^{\circ}$ long around $20^{\circ} \mathrm{W}$, and averaged over lat bins of $2.5^{\circ}$ and potential temperature bins of $50 \mathrm{~K}$. 


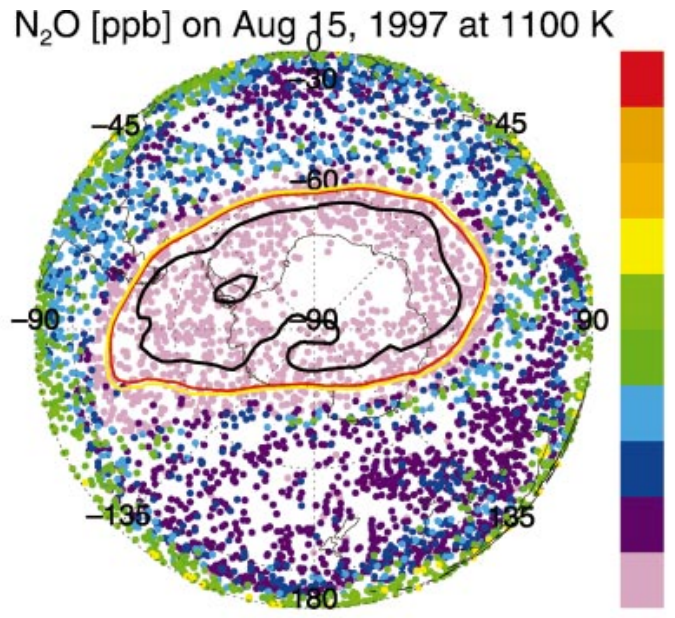

180.00

160.00

140.00

120.00

100.00

80.00

60.00

40.00

20.00

0.00

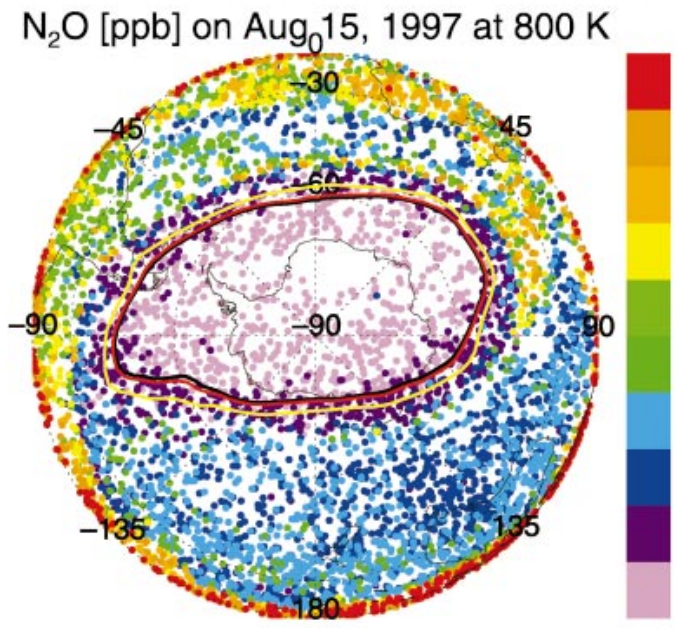

219.20

180.00

160.00

140.00

120.00

100.00

80.00

60.00

40.00

20.00

0.00

FIG. 4. Horizontal cross sections through the Antarctic polar vortex on 15 Aug 1997 at 1200 UTC. CRISTA measurements of $\mathrm{N}_{2} \mathrm{O}$ for (top) $1100( \pm 25) \mathrm{K}$ and (bottom) $800( \pm 25) \mathrm{K}$. The data for 15 Aug 1997 were constructed by trajectory mapping of all CRISTA measurements since 8 Aug forward to 15 Aug 1997, 1200 UTC. Also shown is the vortex boundary defined by the Nash et al. (1996) criterion (red line), as well as the vortex boundary defined by $\Pi_{L}=$ -44.3 PVU (thick black line) and defined by $\Pi_{g}=-44.3$ (with $\varepsilon$ $=4$; yellow line).

subtropical air, rich in $\mathrm{N}_{2} \mathrm{O}$. These tongues are drawn around the vortex due to a perturbation of the vortex by a planetary wave of zonal wavenumber 2 (Günther et al. 2002)

\section{Discussion}

\section{a. The choice of the polytropic exponent $\varepsilon$}

For the analysis of a particular meteorological situation, the parameter $\varepsilon$ in the formulation of the generalized modified potential vorticity $\Pi_{g}$ [cf. Eq. (11)] should be chosen so that Eq. (4) describes approximately the temperature profile in question. Similar to an earlier polar vortex study (Nakamura 1995), we have concluded that choosing $\varepsilon=4$ results in the best representation of the polar vortex over the complete altitude range considered here (i.e., 350-1400 K). However, were one to focus solely on lower altitudes, below $\approx 800 \mathrm{~K}$, where a late winter high-latitude temperature profile would be approximately isothermic, an isothermic scaling $(\varepsilon=$ 4.5) would describe the vertical vortex structure more accurately. Further, comparisons like the one shown in Fig. 2 are influenced by the choice of the reference level $\theta_{0}$. Finally, one might envisage even more sophisticated scalings, where a different $\varepsilon$ is used above and below a suitably chosen reference level $\theta_{0}$, for which the different scaling factors would agree.

\section{b. Equivalent latitude versus $\Pi_{g}$ as a quasi- meridional coordinate}

The natural meridional coordinate, latitude, has the distinct disadvantage that is does not constitute the boundary of an air mass (in a Lagrangian sense) if followed over a certain range of longitudes and even more so for a zonal average. To obviate this problem, a number of so-called equivalent latitudes were suggested that consider the area $A$ enclosed by the contour along a given value of a certain quasi-conservative tracer as a meridional coordinate. The equivalent latitude is then defined as the limiting latitude if $A$ were reshaped into a circle centered around the pole. Possible tracers are potential vorticity (Butchart and Remsberg 1986; Nakamura 1995), the mixing ratio of long-lived trace gases (Nakamura and Ma 1997), or artificial tracers that may be advected in model calculations (Haynes and Shuckburgh 2000; Allen and Nakamura 2003).

Potential vorticity itself could serve as a quasi-meridional coordinate, were it not for its exponential increase with altitude. However, $\Pi_{g}$ can be constructed in a way such that it remains approximately constant with altitude and may thus serve as an alternative diagnostic tool to equivalent latitude in certain applications. While equivalent latitude is more intuitive, an analysis employing $\Pi_{g}$ would avoid the additional step of calculating areas enclosed by given tracer contours.

\section{c. Absolute vorticity as an alternative to scaling of potential vorticity}

The problem addressed by Lait (1994) and in this work - that the application of diagnostic tools to the dynamical structure of, for example, the polar vortex over a greater altitude range is hampered by the fact that density and pressure decay exponentially with height in the atmosphere-likewise occurs in the work of Nakamura (1995). He formulates a mass equivalent latitude as a diagnostic tool to both describe the distance of a point from the center of the polar vortex and to identify the boundary of the polar vortex. This is achieved by employing the mass enclosed by contours of a quasi-conservative tracer (like, e.g., $\mathrm{N}_{2} \mathrm{O}$ ) on an isentropic surface as a quasi-meridional coordinate. This 


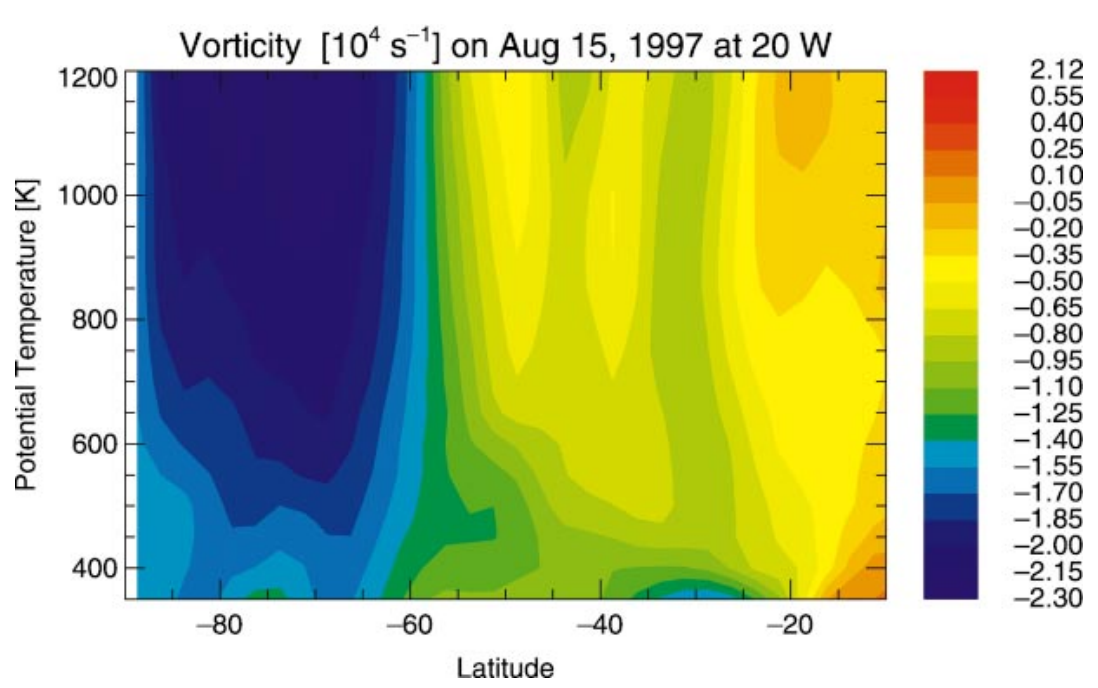

FIG. 5. As in Fig. 3, but showing absolute vorticity $\left(\zeta_{\theta}+f\right)$.

quantity is weighted by a factor $\left(\theta / \theta_{0}\right)^{-\varepsilon}$ to compensate for the exponential decay of density with altitude (Nakamura 1995).

Alternatively, if the divergent component of the isentropic wind $\Delta$ is assumed to vanish, which may be a reasonable assumption in the stratosphere (e.g., McIntyre and Norton 2000, and references therein), then the isentropic density $\sigma$ is constant (because $d \sigma / d t=-\sigma \Delta$ ) so that it is possible to define an area coordinate as a measure of the distance from the center of the polar vortex that does not require weighting to compensate for the density decay with altitude (Nakamura 1996; Nakamura and Ma 1997). These circumstances are analogous to those in question here. For $\Delta=0$ the isentropic density $\sigma=-(g \partial \theta / \partial p)^{-1}$ is constant and thus $\partial \theta / \partial p$ is constant so that potential vorticity may be replaced by absolute vorticity $\left(\zeta_{\theta}+f\right)$ as a diagnostic tool for, for example, the structure of the polar vortex. Indeed, employing $\left(\zeta_{\theta}\right.$ $+f$ ) as a diagnostic tool (Fig. 5), one obtains a good representation of the polar vortex, comparable to that achieved using $\Pi_{L}$ and $\Pi_{g}$ (Fig. 3). However, the absolute vorticity does not lose intensity above $800 \mathrm{~K}$ in contrast to both $\Pi_{L}$ and $\Pi_{g}$. If $\varepsilon=7 / 2$ is chosen to compensate for the rather large value of $\partial \theta / \partial p$ in winter over Antarctica, a $\Pi_{g}$ pattern emerges (not shown) that is rather similar to that of absolute vorticity (Fig. 5).

\section{Conclusions}

We have presented a generalized form $\Pi_{g}$ of Lait's (1994) modified potential vorticity $\Pi_{L}$. The modified potential vorticity $\Pi_{L}$ is obtained from the classic potential vorticity $P$ by introducing a scaling that removes much of the altitude dependence of $P$. The generalization proposed here was derived by allowing for a more general, nonisothermic scaling [Eq. (11)]. The conservation properties of the classic potential vorticity $P$ and of the modified PV $\Pi_{L}$ carry over to $\Pi_{g}$. Further, $\Pi_{g}$ shares the feature of $\Pi_{L}$ to largely remove the exponential increase with height from PV.

Fields of $\Pi_{L}$ and $\Pi_{g}$ were compared with polar vortex edge measures based on both the potential vorticity gradient and measurements of the long-lived trace gas $\mathrm{N}_{2} \mathrm{O}$ by CRISTA. In both cases a constant value of $\Pi_{g}$ describes the vortex edge over a large altitude range to a reasonable approximation, which makes $\Pi_{g}$ a more intuitively interpretable quantity than $\Pi_{L}$.

In summary, through the additional degree of freedom introduced in the definition of the generalized modified potential vorticity $\Pi_{g}$, all desirable properties of Lait's (1994) modified potential vorticity $\Pi_{L}$ are preserved, although this definition allows $\Pi_{g}$ to be adjusted more precisely to the specific problem under investigation.

Acknowledgments. The authors thank L. Lait, N. Nakamura, and an anonymous reviewer for very helpful reviews of an earlier version of this paper. We also thank W. Kouker for comments. Further, we thank D. Offermann and M. Riese for providing the CRISTA-2 data and gratefully acknowledge the efforts of the entire CRISTA team in producing a high-quality dataset. We thank E. Nash for providing his code to determine the vortex edge from potential vorticity fields and for helpful comments on its use. We also thank the UKMO for providing meteorological analyses.

\section{REFERENCES}

Allen, D., and N. Nakamura, 2001: A seasonal climatology of effective diffusivity in the stratosphere. J. Geophys. Res., 106, 7917-7935.

— for isentropic transport studies. J. Atmos. Sci., 60, 287-304.

Butchart, N., and E. E. Remsberg, 1986: The area of the stratospheric polar vortex as a diagnostic for tracer transport on an isentropic surface. J. Atmos. Sci., 43, 1319-1339.

Chandra, S., E. L. Fleming, M. R. Schoeberl, and J. J. Barnett, 1990: 
Monthly mean global climatology of temperature, wind, geopotential height and pressure for 0-120 km. Upper Atmosphere Models and Research, M. Rycroft, G. Keating, and D. Rees, Eds., Advances in Space Research, Vol. 10, 3-12.

Clough, S. A., N. S. Grahame, and A. O'Neill, 1985: Potential vorticity in the stratosphere derived using data from satellites. Quart. J. Roy. Meteor. Soc., 111, 335-358.

Dameris, M., M. Wirth, W. Renger, and V. Grewe, 1995: Definition of the polar vortex edge by LIDAR data of the stratospheric aerosol: A comparison with values of potential vorticity. Beitr. Phys. Atmos., 68, 113-119.

Dunkerton, T. J., and D. P. Delisi, 1986: Evolution of potential vorticity in the winter stratosphere of January-February 1979. $J$. Geophys. Res., 91, 1199-1208.

Ertel, H., 1942a: Ein neuer hydrodynamischer Erhaltungssatz. Naturwissenschaften, 30, 543-544.

_ 1942 b: Ein neuer hydrodynamischer Wirbelsatz. Meteor. Z., 59, 277-281.

Greenblatt, J., and Coauthors, 2002: Defining the polar vortex edge from an $\mathrm{N}_{2} \mathrm{O}$ : Potential temperature correlation. J. Geophys. Res., 107, 8268, doi:10.1029/2001JD000575.

Günther, G., D. S. McKenna, and R. Spang, 2002: The meteorological conditions of the stratosphere for the CRISTA 2 campaign, August 1997. J. Geophys. Res., 107, 8184, doi:10.1029/ 2001JD000692.

Hartmann, D., 1977: On potential vorticity and transport in the stratosphere. J. Atmos. Sci., 34, 968-977.

Haynes, P., and M. McIntyre, 1990: On the conservation and impermeability theorems for potential vorticity. J. Atmos. Sci., 47, 2021-2031.

- , and E. Shuckburgh, 2000: Effective diffusivity as a diagnostic of atmospheric transport, 1: Stratosphere. J. Geophys. Res., 105, $22777-22794$.

Koh, T.-Y., and B. Legras, 2002: Hyperbolic lines and the stratospheric polar vortex. Chaos, 12, 382-394.

Konopka, P., J. U. Grooß, G. Günther, D. S. McKenna, R. Müller, J. W. Elkins, D. Fahey, and P. Popp, 2003: Weak impact of mixing on chlorine deactivation during SOLVE/THESEO 2000: Lagrangian modeling (CLaMS) versus ER-2 in situ observations. J. Geophys. Res., 108, 8324, doi:10.1029/2001JD000876.

Kouker, W., D. Offermann, V. Küll, T. Reddmann, R. Ruhnke, and A. Franzen, 1999: Streamers observed by the CRISTA experiment and simulated in the KASIMA model. J. Geophys. Res. 104, $16405-16418$

Lait, L. R., 1994: An alternative form for potential vorticity. J. Atmos. Sci., 51, 1754-1759.

Lary, D. J., M. P. Chipperfield, J. A. Pyle, W. A. Norton, and L. P. Riishøjgaard, 1995: Three-dimensional tracer initialization and general diagnostics using equivalent PV latitude-potential-temperature coordinates. Quart. J. Roy. Meteor. Soc., 121, 187-210.

Leovy, C. B., C.-R. Sun, M. H. Hitchman, E. E. Remsberg, J. M. Russell III, L. L. Gordley, J. C. Gille, and L. V. Lyjak, 1985: Transport of ozone in the middle stratosphere: Evidence for planetary wave breaking. J. Atmos. Sci., 42, 230-244.

Manney, G. L., R. W. Zurek, A. O’Neill, and R. Swinbank, 1994 On the motion of air through the stratospheric polar vortex. $J$. Atmos. Sci., 51, 2973-2994.

_ R. Swinbank, and A. O'Neill, 1996: Stratospheric meteorological conditions for the 3-12 Nov 1994 ATMOS/ATLAS 3 measurements. Geophys. Res. Lett., 23, 2409-2412.

, H. Michelsen, M. L. Santee, M. Gunson, F. Irion, A. Roche, and N. Livesey, 1999: Polar vortex dynamics during spring and fall diagnosed using trace gas observations from the Atmospheric Trace Molecule Spectroscopy instument. J. Geophys. Res., 104, 18 841-18 866.
McIntyre, M. E., 1992: Atmospheric dynamics: Some fundamentals, with observational implications. Proceeding of the International School of Physics "Enrico Fermi" CXV Course, J. C. Gille and G. Visconti, Eds., North-Holland, 313-386.

- and W. A. Norton, 2000: Potential vorticity inversion on a hemisphere. J. Atmos. Sci., 57, 1214-1235; Corrigendum, 58, 949.

McKenna, D. S., P. Konopka, J.-U. Grooß, G. Günther, R. Müller, R. Spang, D. Offermann, and Y. Orsolini, 2002a: A new Chemical Lagrangian Model of the Stratosphere (CLaMS). Part I: Formulation of advection and mixing. J. Geophys. Res., 107, 4309, doi:10.1029/2000JD000114

_, J.-U. Grooß, G. Günther, P. Konopka, R. Müller, G. Carver, and Y. Sasano, 2002b: A new Chemical Lagrangian Model of the Stratosphere (CLaMS). Part II: Formulation of chemistryscheme and initialisation. J. Geophys. Res., 107, 4256, doi: 10.1029/2000JD000113.

Morgenstern, O., and G. Carver, 2001: Comparison of cross-tropopause transport and ozone in the upper troposphere and lower stratosphere region. J. Geophys. Res., 106, 10 205-10 221.

Morris, G. A., and Coauthors, 1995: Trajectory mapping and applications to data from the Upper Atmosphere Research Satellite. J. Geophys. Res., 100, 16 491-16 505.

Nakamura, N., 1995: Modified Lagrangian-mean diagnostics of the stratospheric polar vortices. Part I: Formulation and analysis of GFDL SKYHI GCM. J. Atmos. Sci., 52, 2096-2108.

_ 1996: Two-dimensional mixing, edge formation, and permeability diagnosed in area coordinates. J. Atmos. Sci., 53, 1524 1537.

_, and J. Ma, 1997: Modified Lagrangian-mean diagnostics of the stratospheric polar vortices, 2: Nitrous oxide and seasonal barrier migration in the cryogenic limb array etalon spectrometer and SKYHI general circulation model. J. Geophys. Res., 102, 25 72125735 .

Nash, E. R., P. A. Newman, J. E. Rosenfield, and M. R. Schoeberl, 1996: An objective determination of the polar vortex using Ertel's potential vorticity. J. Geophys. Res., 101, 9471-9478.

Offermann, D., K.-U. Grossmann, P. Barthol, P. Knieling, M. Riese, and R. Trant, 1999: Cryogenic Infrared Spectrometers and Telescopes for the Atmosphere (CRISTA) experiment and middle atmosphere variability. J. Geophys. Res., 104, 16 311-16 325.

Polvani, L., and R. Saravanan, 2000: The three-dimensional structure of breaking Rossby waves in the polar wintertime stratosphere. J. Atmos. Sci., 57, 3663-3685.

Rex, M., and Coauthors, 1998: In situ measurements of stratospheric ozone depletion rates in the Arctic winter 1991/92: A Lagrangian approach. J. Geophys. Res., 103, 5843-5853.

Riese, M., R. Spang, P. Preusse, M. Ern, M. Jarisch, D. Offermann, and K. Grossmann, 1999: Cryogenic infrared spectrometers and telescopes for the atmosphere (CRISTA) data processing and atmospheric temperature and trace gas retrieval. J. Geophys. Res., 104, 16 349-16 367.

Rummukainen, M., B. Knudsen, and P. von der Gathen, 1994: Dynamical diagnostics of the edges of the polar vortices. Ann. Geophys., 12, 1114-1118.

Schoeberl, M. R., L. R. Lait, P. A. Newman, and J. E. Rosenfield, 1992: The structure of the polar vortex. J. Geophys. Res., 97, 7859-7882.

- and Coauthors, 1996: Development of the Antarctic ozone hole. J. Geophys. Res., 101, 20 909-20 924.

Tuck, A. F., 1989: Synoptic and chemical evolution of the Antarctic vortex in late winter and early spring, 1987. J. Geophys. Res., 94, $11687-11737$.

Waugh, D., and W. Randel, 1999: Climatology of Arctic and Antarctic polar vortices using elliptical diagnostics. J. Atmos. Sci., 56, $1594-1613$ 